\title{
A Latent Analysis of Earth Surface Dynamic Evolution Using Change Map Time Series
}

\author{
Corina Văduva, Teodor Costăchioiu, Carmen Pătraşcu, Inge Gavăt, Senior Member, IEEE, \\ Vasile Lăzărescu, Member, IEEE, and Mihai Datcu, Senior Member, IEEE
}

\begin{abstract}
With a continuous increase in the number of Earth Observation satellites, leading to the development of satellite image time series (SITS), the number of algorithms for land cover analysis and monitoring has greatly expanded. This paper offers a new perspective in dynamic classification for SITS. Four similarity measures (correlation coefficient, Kullback-Leibler divergence, conditional information, and normalized compression distance) based on consecutive image pairs from the data are employed. These measures employ linear dependences, statistical measures, and spatial relationships to compute radiometric, spectral, and texture changes that offer a description for the multitemporal behavior of the SITS. During this process, the original SITS is converted to a change map time series (CMTS), which removes the static information from the data set. The CMTS is analyzed using a latent Dirichlet allocation (LDA) model capable of discovering classes with semantic meaning based on the latent information hidden in the scene. This statistical method was originally used for text classification, thus requiring a word, document, corpus analogy with the elements inside the image. The experimental results were computed using 11 Landsat images over the city of Bucharest and surrounding areas. The LDA model enables us to discover a wide range of scene evolution classes based on the various dynamic behaviors of the land cover. The results are compared with the Corinne Land Cover map. However, this is not a validation method but one that adds static knowledge about the general usage of the analyzed area. In order to help the interpretation of the results, we use several studies on forms of relief, weather forecast, and very high resolution images that can explain the wide range of structures responsible for influencing the dynamic inside the resolution cell.
\end{abstract}

Manuscript received February 29, 2012; revised June 15, 2012; accepted August 26, 2012. Date of publication November 20, 2012; date of current version March 21, 2013. This work was supported in part by the Sectoral Operational Program Human Resources Development 2007-2013 of the Romanian Ministry of Labour, Family and Social Protection through the Financial Agreement POSDRU/107/1.5/S/76903.

C. Văduva, T. Costăchioiu, C. Pătraşcu, I. Gavăt, and V. Lăzărescu are with the Research Center for Spatial Information, Department of Applied Electronics and Information Engineering, Faculty of Electronics, Telecommunications and Information Technology (ETTI), University "Politehnica" of Bucharest, 061071 Bucharest, Romania (e-mail: corina.vaduva@gmail.com; c_teodor@yahoo.com; cami.patrascu@gmail.com; igavat@lpsv.pub.ro; vl@elia.pub.ro).

M. Datcu is with the Remote Sensing Technology Institute, German Aerospace Center (DLR), 82234 Oberpfaffenhofen, Germany. He is also with the Research Center for Spatial Information, Department of Applied Electronics and Information Engineering, Faculty of Electronics, Telecommunications and Information Technology, University "Politehnica" of Bucharest, 061071 Bucharest, Romania (e-mail: mihai.datcu@dlr.de).

Color versions of one or more of the figures in this paper are available online at http://ieeexplore.ieee.org.

Digital Object Identifier 10.1109/TGRS.2012.2219316
Index Terms-Change detection, dynamic evolution classification, latent Dirichlet allocation (LDA) model, satellite image time series (SITS), similarity measures.

\section{INTRODUCTION}

$\mathbf{T}$ HE Earth's surface is constantly affected by various processes, thus explaining why the study of its dynamics is one of the emergent issues in the field of remote sensing imagery processing. Seasons, climate, natural disasters, human activities, and the urban development are few of the reasons underlying surface transformations. Monitoring the land cover evolution is imperative for understanding the environmental changes and complex land transformations identifiable over short (days-weeks) to long (seasonal-years) periods of time. Vast data collections have been created using images presenting a region at different moments of time (years, months, or days), which have been identified as satellite image time series (SITS) that can provide significant knowledge about the Earth's surface dynamics.

By exploiting their rich information content, a broad range of new applications may be opened. For instance, by using SITS covering short periods of time, one can observe the growth and the maturation periods of cultures and their harvesting, the evolution of rivers and flooded areas, or other details helpful in domains such as agriculture, forestry, or hydrology. Moreover, images acquired during long periods of time may explain urban development, natural resources exploitation and their subsequent consequences, or the pollution phenomenon. These are only few examples of potential applications for urbanization, industry, and ecology.

It is difficult though for the human eye to observe and extract relevant information directly from such an important amount of data. Therefore, significant attention has been paid to a set of various techniques developed for temporal information extraction [1] in order to apply them on SITS. Several methods have been developed for the automatic discovery of regularities or relationships inside collections of unstructured data indexed according to the temporal interdependences. The spatiotemporal study presented in [2] aims at extracting relevant information based on the information-bottleneck principle. A method depending on a suitable model selection and a ratedistortion analysis are combined. A novel method for extraction of frequent temporal patterns is depicted in [3]. It relies on the identification of evolutions and subevolutions at pixel level for finding groups of pixels that could be of interest to the end users. Furthermore, unsupervised learning of dynamic cluster trajectories followed by an interactive learning 
process is proposed in [4]. Landsat SITS was characterized considering spectral indices [5] and a mixed information measure [6]. A methodology for compressing an image database is presented in [19] by taking into account interobject redundancies and using the informational similarity measure. Recently, a method for SITS analysis that is able to deal with temporal irregular sampling and to compare time series having different numbers of temporal observations based on dynamic-time-warping similarity measure has been introduced in [27].

Nevertheless, the scene dynamic can be analyzed considering only two images and computing how alike they are. This could be extremely helpful for finding specific details about the transformations that a certain area had suffered at a specific moment of time. In order to identify those transformations, change detection algorithms may be applied on image pairs depicting the same region at different moments of time, before and after a certain event. Similarity measures are generally used to estimate the degree of variation between the pixels of two images. The correlation coefficient (CC) is probably one of the most common similarity measures, and it helps highlighting radiometric changes [7], [8]. The Kullback-Leibler divergence (KLD) [8] and the conditional information (CI) [9] are two measures that emphasize the changes associated to the spectral features of the compared scenes. The changes related to the images' texture are captured when computing the normalized compression distance (NCD) [10], [11]. While change detection algorithms using two images represent the starting point for long-term analysis, leading to SITS modeling, the similarity measures employed provide new features for describing the evolution of scene dynamics.

Supporting this idea, this paper introduces a new approach for the study of land cover dynamic evolution by combining SITS temporal analysis with classical change detection techniques. The main idea is to model an image time series based on the transformations occurring between consecutive acquisitions of the considered SITS. The innovation of the proposed technique lies in the conversion of the multispectral information from the entire data set in a change map time series (CMTS). Hereby, each pair of consecutive images will be described by a number of four change maps computed using the four different similarity measures mentioned previously. Complementary information about the changes occurred in the scene will be extracted and further employed in order to provide the user with a broader perspective on the land transformation processes.

CMTS size will quadruple compared to the original SITS, making the usual processing even more difficult. In order to structure the collection of images for a fast analysis, each pixel of the scene will be described by a temporal signature. A $k$-means classification will be performed over all available signatures, such that the values assigned to a precise position in the image will be reduced to a single label and denoted as a "word." In order to apply text statistics for image analysis, the definition of a dictionary is mandatory. For this purpose, the authors propose a $k$-means classification over the set of temporal signatures (words) such that the assigned labels denote the dynamic evolution of pixels in the scene. Moreover, each class can be regarded as a "visual word," and it comprises areas characterized by the same type of change in the same period of time as the SITS. For instance, different types of crops or vegetation can be separated according to their periodicity and life spans. Based on this image-text analogy, the scene described by the CMTS will be modeled using latent Dirichlet allocation (LDA) generative model [12], [13], a technique developed for text analysis. Using a bag-of-words assumption, the order of visual words is neglected, and the dictionary will be reduced to a set of latent parameters treated as variables drawn from a Dirichlet distribution. The result is a thematic map which highlights regions with similar dynamic evolution, offering a different perspective from the already existing ones.

All the previously developed algorithms for SITS modeling analyze sequences of image classifications characterizing the scene at the time of data acquisition. Therefore, the process uses precisely defined temporal attributes as inputs to obtain a classification of the considered scene. The evolution of Earth surface is indirectly pursued.

The methodology that we propose in this paper emphasizes the process of Earth surface dynamic evolution using attributes of change which are computed in order to express the land dynamic between consecutive image acquisitions. Based on these attributes, the generative process of LDA model is able to latently discover classes of evolution whose semantics is defined by similar dynamic evolution in time of different regions. The LDA modeling proved to be effective with a small training data set, randomly chosen and with no prior information about the classes to be extracted. Even if our methodology may seem similar to a classification process, LDA is nevertheless an algorithm for information retrieval. In this case, it can find, annotate, and return regions characterized by a similar evolution in time, pointing toward specific directions of Earth surface transformation.

The remainder of this paper is organized as follows. Section II presents the method proposed by the authors for the analysis of Earth surface dynamic evolution. The similarity measures used to describe short-time changes (CC, KLD, CI, and NCD) are defined in Section III, while the LDA modeling of the dynamic evolution is explained in Section IV. Experimental results (Section V) and the conclusions (Section VI) will contribute to the exemplification and validation of the proposed methodology.

\section{Proposed Method For the Latent Analysis OF EARTH SURFACE DYNAMIC EVOLUTION}

This section focuses on describing the proposed methodology for the analysis of Earth surface dynamic using remote sensing image time series.

Considering a SITS with $N+1$ images (of $m \times p$ pixels each), we form pairs of consecutive acquisitions that will be integrated as inputs of a change detection process. Four different similarity measures will be used to identify various types of changes occurred in the scene, offering the user an overview about the nature of the analyzed landscape. The four different measures will provide complementary information as follows: the $\mathrm{CC}$ determines radiometric changes, the KLD 


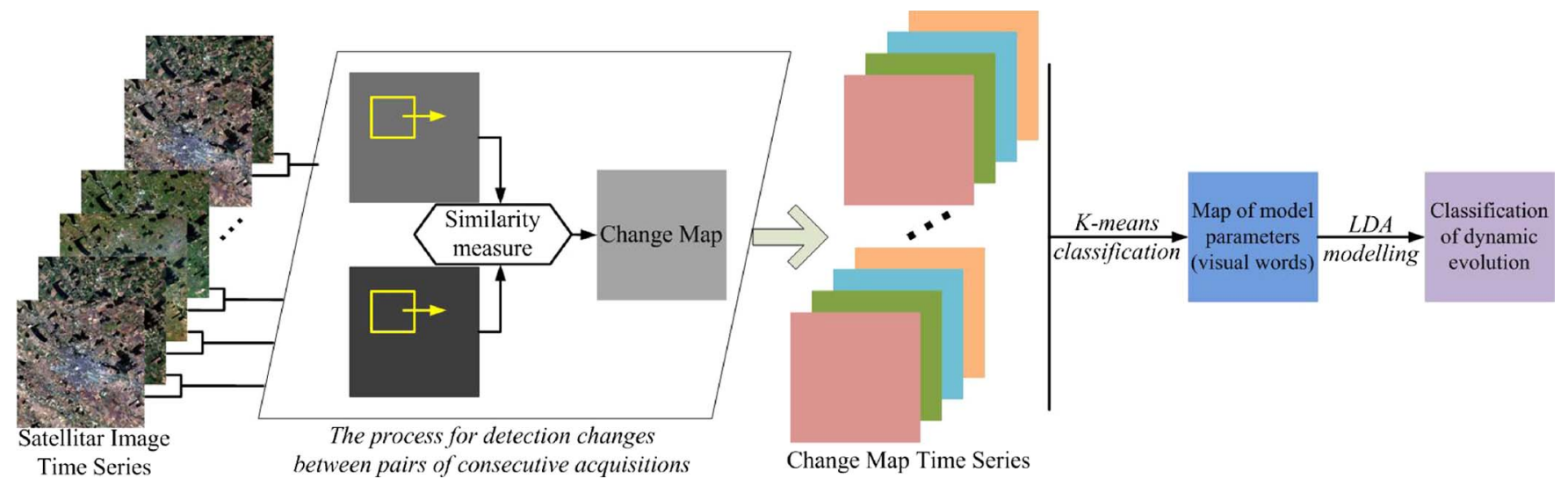

Fig. 1. Processing chain of the proposed approach used to classify the dynamic evolution of the scene.

and CI measure the local and, respectively, general spectral differences, and the NCD is used for detecting transformations of the land cover texture.

In order to perform a change detection analysis, we employ a sliding window that simultaneously covers the same area from both images. The size of the window depends on both the image spatial resolution and the type of analyzed change. The value of the similarity measure gives us the amount of change corresponding to the pixel in the center of each tile. The resulting change maps are gray level images with the intensity of the pixel defining the degree of change. Thereby, each pair of consecutive acquisitions will be characterized by a set of four change maps.

The output of the change detection process consists of a series of $4^{*} N$ components comprising four change maps computed based on the similarity measures employed for each of the $N$ pairs of images in the original SITS consecutively acquired. A new quadruple sized time series is formed based on the newly computed maps. At this stage, every pixel will be described by a $4^{*} N$ component temporal signature that depicts the dynamic evolution of the area.

Once the CMTS is obtained, a $k$-means algorithm employing a Euclidean distance will be applied on the set of $m \times p$ temporal signatures obtained in order to define some patterns for the dynamic evolution. All the pixels characterized by a similar temporal behavior will receive the same label, reducing the CMTS to a single image. These patterns will be denoted as visual words and used as inputs for the LDA modeling in order to perform an interactive classification which is based on a text analysis method. Considering that LDA is a probabilistic generative model for collections of discrete data or text corpora, the purpose is to identify symbolic characteristics for the objects in the analyzed collection that enable efficient processing while preserving fundamental statistical relationships necessary for a semantic classification of Earth's surface dynamic. The model requires an arbitrary training set to learn the statistic interactions in the scene and afterward apply them for the analysis of the entire collection. The scene will be described as a mixture of topics characterized by latent variables. Every topic is, in turn, distinguished by means of a probability distribution based on the co-occurrence of visual words extracted from the image.
A general diagram for the proposed approach is shown in Fig. 1. The method is unsupervised and assigns semantic meaning to the scene, according to the dynamic evolution of the Earth's surface.

Nevertheless, the extension of the SITS with a new image implies the resumption of the entire analysis, because no new information can be added without discarding the previous model. After the radiometric correction of the new image, depending on its time positioning in the series, one or two sets of change maps need to be obtained and then introduced in the CMTS. Even if this is a simple and independent computation, it will significantly affect the parameters of the analysis. This will result in a new feature space, and for vector quantization, we have to apply $k$-means on this new feature space. Increasing the dimensionality of the feature space also changes the semantic meaning of the extracted "words," and as such, the optimal number of LDA topics and the optimal number of words have to be determined. The final influence depends on the amount of existing differences between the new scene and the existing ones.

A theoretical overview over the similarity measures and the LDA generative model is given in the following sections, detailing the computation method and type of information that it extracts from a SITS in order to help environmental understanding based on Earth's land cover dynamic.

\section{Similarity Measures for Change Detection}

During the change detection process, we compare two images acquired at different times, before and after an unspecified event, unknown for the user. The purpose is to identify the differences occurred in the landscape by applying a patchbased analysis on two images of the same size. Therefore, we simultaneously scan the images with a window, measure the similarity between them, and assign the obtained value to the coordinates corresponding to the center of the window. The measures applied assess similarity from different points of view. Thus, the CC computes radiometric information based on linear dependences, the KLD and CI use first- and second-order statistics to depict spectral changes, and the NCD considers the spatial information to extract textural differences between two regions in consecutive images. 


\section{A. $C C$}

The $\mathrm{CC}$ is a measure of the dependences existing between two quantities, and we use it to express the quality of a least squares fitting of the image after to the one before a certain event.

The computation of the $\mathrm{CC}$ between two image windows $i$ (in the first image) and $j$ (in the second image) can be made by means of the following formula:

$$
\mathrm{CC}(i, j)=\frac{1}{n} \frac{\sum_{x, y}\left(i(x, y)-m_{i}\right)\left(j(x, y)-m_{j}\right)}{\sigma_{i} \sigma_{j}}
$$

where $n$ is the number of pixels included in the analysis window, $x$ and $y$ represent their coordinates, $m_{i}$ and $m_{j}$ are the estimated mean values for the $i$ th and $j$ th windows, respectively, and $\sigma_{i}$ and $\sigma_{j}$ are their standard deviations. $\operatorname{CC}(i, j)=0$ when the landscape is completely changed, while $\mathrm{CC}(i, j)=1$ for identical images.

There are two approaches for the use of this similarity measure; it can model geometric deformations by local rigid displacements [7] or detect radiometric scene transformations [8]. In this paper, the authors employ the CC in order to identify radiometric changes occurred between two consecutive images included in a SITS.

\section{B. First-Order KLD}

The KLD is based on pixel statistics and has been used as a similarity measure for change or anomaly detection processes in image analysis [7], [8], [11]. In this paper, we apply this measure to detect the transformations that occurred in the landscape, aiming to quantify the divergence between two image windows characterized by the probability distributions $p(x)$ (in the first image) and $q(x)$ (in the second image).

Considering the two probability distributions $p(x)$ and $q(x)$ related to the same random variable $X$, the KLD or relative entropy [14] is given by

$$
D_{\mathrm{KL}}(p, q)=\sum_{x} p(x) \log \frac{p(x)}{q(x)} .
$$

The KLD is a positively defined not-symmetric measure, being equal to zero only if $p(x)=q(x)$. When the analyzed images are very similar, $D_{\mathrm{KL}}(p, q) \rightarrow 0$.

Based on this measure, the change detection algorithm compares the density probabilities of the gray level pixels for both image windows, offering information about the spectral differences of the data. It is important to mention that all the pixels inside a sliding window are characterized by identical weights.

\section{C. $C I$}

The third algorithm for change detection is an adapted version of the Alparone method used for synthetic aperture radar images [8]. The rationale is that the negative of logarithm of probability of an amplitude level in one image conditional to the level of the same pixel in the other image conveys information on the amount of change occurred between the two images.
The algorithm is based on the computation of the CI at each pixel $(m, n)$ from the before and after images:

$$
\mathrm{CI}(m, n)=-\log \left(q\left(\left\lfloor\bar{g}_{2}(m, n)\right\rfloor \mid\left\lfloor\bar{g}_{1}(m, n)\right\rfloor\right)\right.
$$

where $g_{1}$ and $g_{2}$ are the before and after images and $q$ is the rescaled conditional probability.

Although this algorithm finds spectral changes between the pair of images, it differs from the KLD by decreasing the weights of the pixels toward the edges of the window. Further advantages of the method are its low sensitivity to noise and lack of preprocessing requirements.

\section{D. $N C D$}

The NCD is a universal parametric free metric successfully applied as a similarity measure to unstructured data in various domains such as text corpora, computer programs, genomes, or images. The NCD considers the length of the shortest binary program used to transform two items into each other [10]. Its main idea is based on the Kolmogorov complexity, a noncomputable notion that needs to be approximated using a compressor (i.e., gzip and jpeg).

In the present paper, we use the NCD to measure the differences between two images and to detect major structural changes that occurred in the landscape. Both spectral and texture transformations will be identified.

The NCD is obtained by employing the following equation, where $i$ and $j$ are the analysis windows in the first and second images, respectively:

$$
\operatorname{NCD}(i, j)=\frac{C(i, j)-\min \{C(i), C(j)\}}{\max \{C(i), C(j)\}} .
$$

$C(i, j)$ denotes the compressed size of the concatenation of $i$ and $j, C(i)$ denotes the compressed size of $i$, and $C(j)$ denotes the compressed size of $j$.

The result is a nonnegative number $0<r<1+e$ representing how different the two images are. Therefore, smaller numbers (darker areas) represent more similar parts in the map of changes. The error $e$ in the upper bound appears due to the imperfections in the compression technique (in our case, the "zip" program).

\section{LDA MODELING OF THE DyNAMIC EVOLUTION}

After we have identified the changes that occurred between consecutive images of the SITS, the CMTS is complete and ready to be modeled in order to assess the dynamic evolution of the Earth land cover. An interactive classification is performed by applying a statistical tool initially developed for text classification [12] and also used for semantic image annotation [13], [15], [16].

This is a generative model for collections of discrete data named LDA, a random source that is able to generate infinite sequences of samples based on a probability distribution [12]. LDA is a three-level hierarchical Bayesian model (word, document, corpus) founded on the bag-of-words assumption, meaning that the order of words inside a document and that 
of documents inside a corpus are ignored. Consequently, each document in the collection is defined as a finite random mixture over a "latent" hidden set of topics, while a topic is defined as a probability distribution over a set of words in the vocabulary.

Denoting a document as a collection of $N$ words in the vocabulary and the vocabulary $W=\left\{w_{1}, w_{2}, \ldots, w_{N}\right\}$ and the corpus as a collection of $M$ documents $D=$ $\left\{W_{1}, W_{2}, \ldots, W_{M}\right\}$, LDA assumes a generative process for each document in the corpus, as will be described hereinafter [12].

First, we choose a $K$-dimensional Dirichlet random variable $\theta \sim \operatorname{Dir}(\alpha)$, where $K$ is considered known and preset. Furthermore, for each of the word positions $n \in\{1,2, \ldots, N\}$, we choose a topic $z_{n} \sim \operatorname{Multinomial}(\theta)$ and a word $w_{n}$ from $p\left(w_{n} \mid z_{n}, \beta\right)$, a multinomial probability conditioned on the topic $z_{n}$. The word probabilities are parameterized by a matrix $\beta_{i j}=$ $p\left(w_{j}=1 \mid z_{i}=1\right)$ whose size is given by the size of the vocabulary and by the dimensionality of the Dirichlet distribution. It is treated as a fixed quantity to be expected.

The corpus level parameters $\alpha$ and $\beta$ are estimated during the training phase, $\theta$ characterizes the documents, and $z$ and $w$ represent the word level variables. Given $\alpha$ and $\beta$, one can compute the joint distribution of a topic mixture $\theta$, a set of $N$ topics $z$, and a set of $N$ words $w$ using the following formula, where $\theta_{i}$ is represented in terms of the $p\left(z_{n} \mid \theta\right)$ probability for the unique $i$ when $z_{n}^{i}=1$ :

$$
p(\theta, z, W \mid \alpha, \beta)=p(\theta \mid \alpha) \prod_{n=1}^{N} p\left(z_{n} \mid \theta\right) p\left(w_{n} \mid z_{n}, \beta\right) .
$$

Taking into consideration all the values of $\theta$ and $z$, we can compute the marginal distribution of a document based on (6). Furthermore, the probability of the corpus is measured as the product of the marginal probabilities of single documents (7)

$$
\begin{aligned}
& p(W \mid \alpha, \beta) \\
& \quad=\int p(\theta \mid \alpha)\left(\prod_{n=1}^{N} \sum_{z_{n}} p\left(z_{n} \mid \theta\right) p\left(w_{n} \mid z_{n}, \beta\right)\right) d \theta \\
& p(D \mid \alpha, \beta) \\
& \quad=\prod_{d=1}^{M} \int p\left(\phi_{d} \mid \alpha\right)\left(\prod_{n=1}^{N_{d}} \sum_{z_{d n}} p\left(z_{d n} \mid \theta_{d}\right) p\left(w_{d n} \mid z_{d n}, \beta\right)\right) d \theta_{d} .
\end{aligned}
$$

We mention that $M$ is the number of documents in the corpus and $N_{d}$ is the number of words in a document. Within the three levels involved by LDA, the documents can be associated with multiple topics. Due to its flexibility to assign probabilities to documents outside the training corpus, LDA is considered a suitable algorithm for supervised classification over an unknown data set.

In order to be able to apply the algorithm to CMTS, we need to define a correspondence between text and the current approach. Due to the fact that the probability of two pixels describing similar time evolutions in terms of change maps is small, we have to perform a $k$-means classification to label visual words and, thus, the vocabulary. Therefore, each pixel characterized by a $4^{*} N$ component temporal signature repre-
TABLE I

ACQUISITION DATES FOR SITS

\begin{tabular}{|l|l|l}
\hline $16 / 05 / 2007$ & $03 / 07 / 2007$ & $20 / 08 / 2007$ \\
\hline $25 / 05 / 2007$ & $19 / 07 / 2007$ & $29 / 08 / 2007$ \\
\hline $10 / 06 / 2007$ & $28 / 07 / 2007$ & $14 / 09 / 2007$ \\
\hline $26 / 06 / 2007$ & $04 / 08 / 2007$ & \\
\hline
\end{tabular}

TABLE II

IMAge PAIRS AND THE CORRESPONDING TIME PERIODS For Change Detection Analysis

\begin{tabular}{|c|c|c|c|}
\hline $\begin{array}{c}\text { Image } \\
\text { pair }\end{array}$ & Time Period & $\begin{array}{c}\text { Image } \\
\text { pair }\end{array}$ & Time Period \\
\hline Ip1 & $16 / 05 / 2007-25 / 05 / 2007$ & Ip6 & $19 / 07 / 2007-28 / 07 / 2007$ \\
\hline Ip2 & $25 / 05 / 2007-10 / 06 / 2007$ & Ip7 & $28 / 07 / 2007-04 / 08 / 2007$ \\
\hline Ip3 & $10 / 06 / 2007-26 / 06 / 2007$ & Ip8 & $04 / 08 / 2007-20 / 08 / 2007$ \\
\hline Ip4 & $26 / 06 / 2007-03 / 07 / 2007$ & Ip9 & $20 / 08 / 2007-29 / 08 / 2007$ \\
\hline Ip5 & $03 / 07 / 2007-19 / 07 / 2007$ & Ip10 & $29 / 08 / 2007-14 / 09 / 2007$ \\
\hline
\end{tabular}

sents a visual word. An image tile represents a visual document, while the corpus is the entire CMTS. As the LDA model can only be applied on discrete data, the $k$-means classification can also be considered as a vector quantization process that transforms the extracted feature space into a word space modeled based on the bag-of-words assumption. The rationale behind the selection of this algorithm is its simplicity and frequent use.

\section{LATENT ANALYSiS OF THE EARTH'S SURFACE DYNAMIC EVOLUTION}

The method was tested on 11 Landsat images [20] acquired over a period of five months between May 5 and September 14, 2007 (Table I). The study area is Bucharest and its surroundings, with each scene being $1500 \times 1100$ pixels in size, which corresponds to a surface of $45 \times 33 \mathrm{~km}$. Table II presents the time periods for the change detection analysis.

\section{A. Experimental Setup}

The proposed processing chain implies the study of a series of 11 images. Even if they are provided by the same sensor, the conditions of acquisition vary according to the Earth-Sun positioning. A data preprocessing is required in order to standardize the SITS for an optimum analysis. The actual methodology described in this paper (Fig. 1) consists of two major parts: change detection algorithm and LDA modeling. Their parameter setup will be presented and detailed within this section along with the characteristics of data preprocessing.

Data Preprocessing: Preprocessing of the data first includes the conversion to the planetary top-of-atmosphere reflectance $\rho_{\lambda}$ [17] using the following equation in order to minimize the illumination effects:

$$
\rho_{\lambda}=\frac{\pi \cdot L_{\lambda} \cdot d^{2}}{\mathrm{ESUN}_{\lambda} \cdot \cos \theta_{s}}
$$




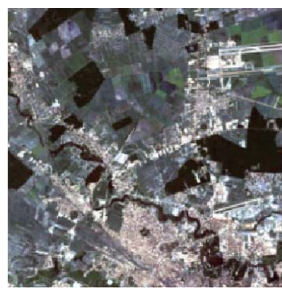

(a)

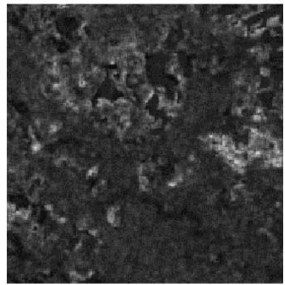

(d)

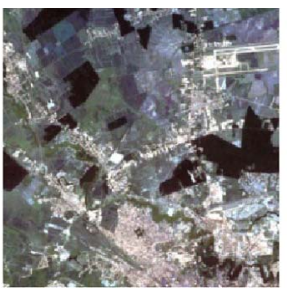

(b)

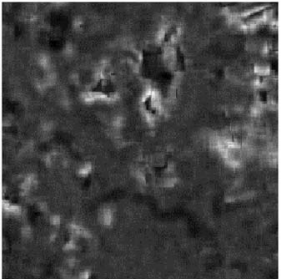

(e)

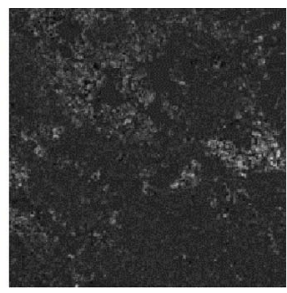

(c)

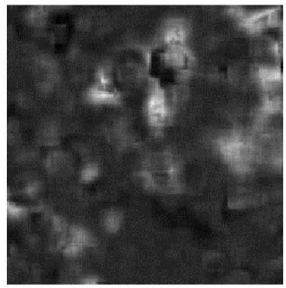

(f)
Fig. 2. (a) and (b) Landsat image $(16.05$, 29.08). (c)-(f) Change maps using the KLD. The sizes of the analysis window are (c) $5 \times 5$ pixels, (d) $10 \times 10$ pixels, (e) $20 \times 20$ pixels, and (f) $30 \times 30$ pixels. As can be noticed, smaller windows increase the sensitivity to local changes.

where $L_{\lambda}$ is the spectral radiance at the sensor's aperture, $d$ is the Earth-Sun distance, $\mathrm{ESUN}_{\lambda}$ is the mean exoatmospheric solar irradiance, and $\theta_{s}$ is the solar zenith angle.

The area chosen for the experiments in this paper is characterized by a temperate climate, with a low occurrence of haze during the studied period. As there are not enough weather data to perform a full radiometric correction, we have performed a relative radiometric correction using the COST method [18], [22], choosing the image from September 19 as reference. Normalization is performed on the basis of an invariant set of pixels by means of a regression analysis. In addition, in order to obtain accurate results, we have selected for the time series analysis only scenes that are cloud free or with a very low presence of clouds.

Every new image needs to be radiometric corrected with respect to the reference scene. There is also the option of setting the new image as reference if it is considered more accurate than the present reference image. If that is the case, a complete radiometric correction will be applied on the entire SITS.

Parameter Setup for Change Detection Process: The extracted classes are then used to compute change maps with the described method: For every pair of consecutive images in the SITS, we simultaneously scan the images with a window, measure the similarity between them, and assign the obtained value to the coordinates corresponding to the center of the window.

The size of the analysis window must be set according to the following three parameters:

1) image spatial resolution - the amount of details one can distinguish;

2) type of deformations desired - the type of change one wants to locate (for instance, a flooded area detection requires a larger window, while damaged houses are identified by a small window);

3) computation time- the computational load of the algorithms increases with the reduction of the window.

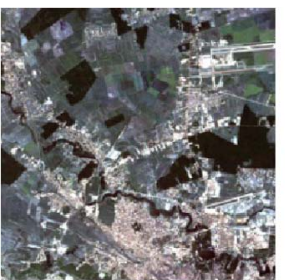

(a)

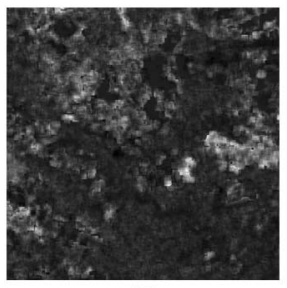

(d)

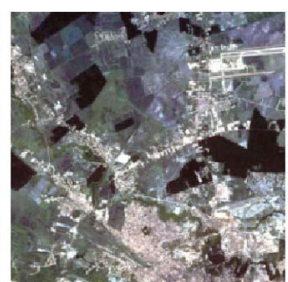

(b)

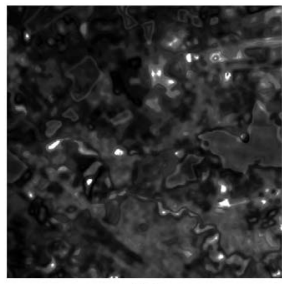

(e)

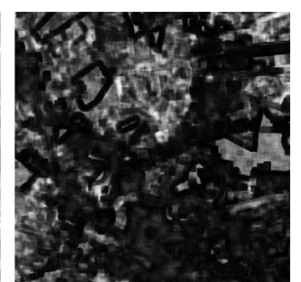

(c)

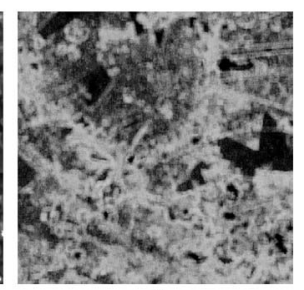

(f)
Fig. 3. (a) and (b) Landsat image $(16.05,29.08)$. (c)-(f) Change maps using CC, KLD, CI, and NCD. Areas with major changes are presented in white, and areas with no changes are presented in black. Changes are visible in different areas due to the complementarity of the extracted features (radiometry, spectral, and texture). The analysis window's size is $10 \times 10$ pixels.

The precision of the method depends significantly on the size of the analysis window, as shown in Fig. 2. The change maps were computed using the KLD similarity measure and a window with various dimensions $(5 \times 5,10 \times 10,20 \times 20$, or $30 \times 30$ pixels).

The change map is a gray level image. Its interpretation depends on the similarity measure used. Consequently, the areas with major changes are colored in white for the KLD, the $\mathrm{CI}$, and the NCD and in black for the CC. In order to uniform the results, we use the negative of the change maps computed based on the CC.

An example illustrating the change maps computed with the four similarity measures for the 16.05-29.08.2007 image pair is shown in Fig. 3.

Considering the employed data, a $10 \times 10$ pixel sliding window was selected experimentally as a compromise between the data features and accuracy of the results. Due to the image low resolution, a window that is too large leads to an important information loss. The identified changes would have no real meaning. Considering these setups, we compute a set of four change maps for every pair of consecutive images in the SITS and form a new image series named CMTS. As a mention, if the original SITS has $N+1$ images, the obtained CMTS has $2 N$ images.

Parameter Setup for LDA Analysis: The LDA modeling is applied on the CMTS. For an accurate analysis, the following two parameters need to be set:

1) number of words;

2) size of the document.

In order to determine these parameters, we propose a method that uses the perplexity to identify an optimum LDA model. The perplexity is equivalent to the inverse of the geometric mean per word likelihood, monotonically decreasing in the likelihood of test data [12]. A good inference extent to the entire data set is given by a low value of this measure. Given a test set of $M$ documents $D=\left\{W_{1}, W_{2}, \ldots, W_{M}\right\}$ containing $N=$ 


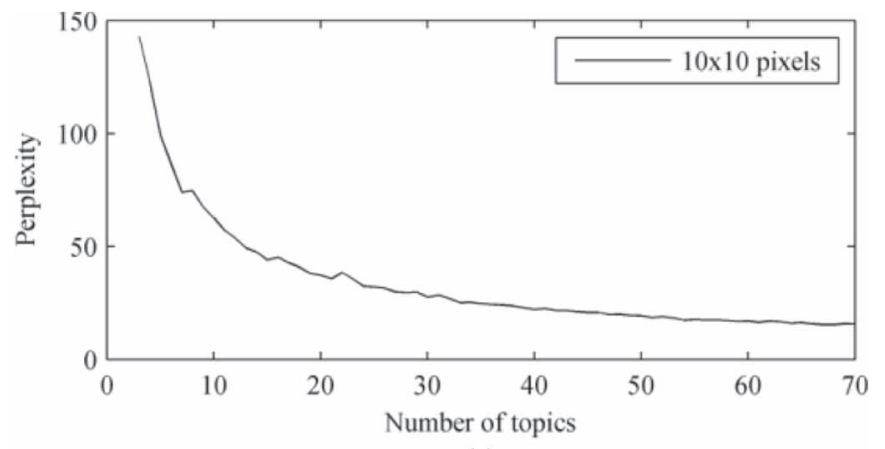

(a)

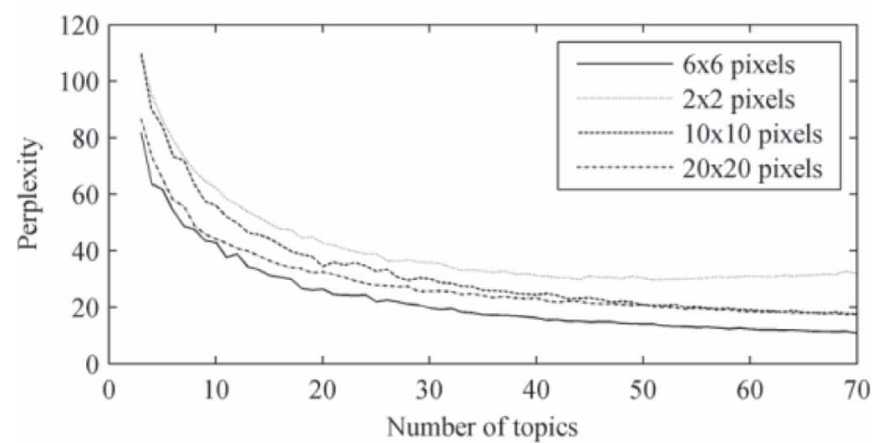

(b)

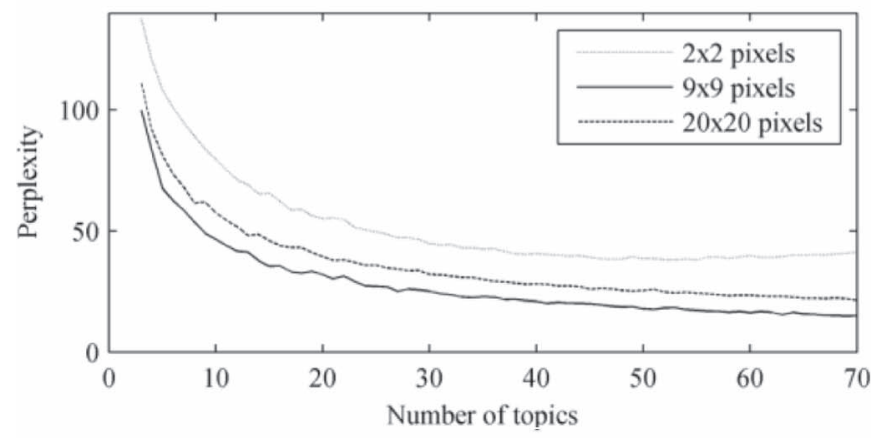

(c)

Fig. 4. Evolution of perplexity based on the document size for dictionaries of 150, 200, and 250 words. (a) Perplexity for 150 words. (b) Perplexity for 200 words. (c) Perplexity for 250 words.

$\left\{N_{1}, N_{2}, \ldots, N_{M}\right\}$ words, the perplexity is computed using the following formula:

$$
\operatorname{perplexity}\left(D_{\text {test }}\right)=e^{-\frac{\sum_{d=1}^{M} \log p\left(W_{d}\right)}{\sum_{d=1}^{M} N_{d}}} .
$$

In our experiments, we considered dictionaries containing 150,200 , and 250 words. For each set of words, we formed documents with variable sizes, and we assessed the perplexity for a number of 3-70 topics. The obtained results are shown in Fig. 4.

We can observe that there is a strong link between the number of words and an optimum size of the document.

1) For the dictionary of 150 words, the optimum size for the document is $10 \times 10$ pixels.

2) For the dictionary of 200 words, the perplexity has the lowest value for a document size of $6 \times 6$ pixels.

3) For the dictionary of 250 words, the optimum size for the document is $9 \times 9$ pixels.

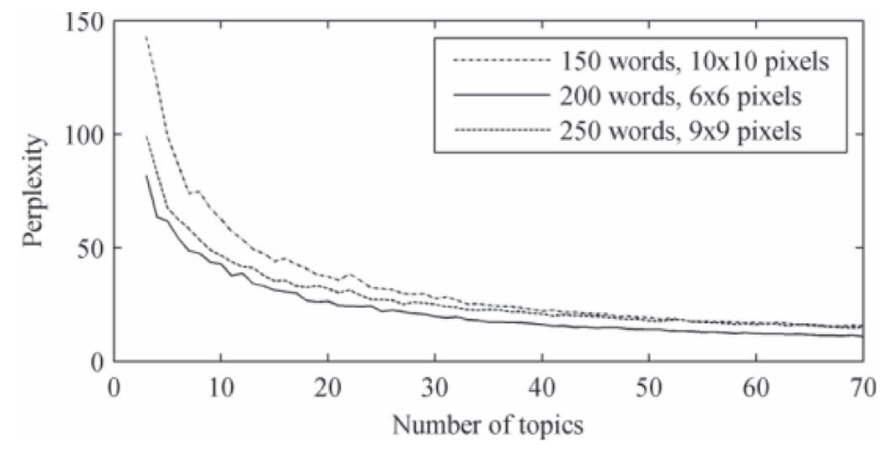

Fig. 5. Perplexity assessment for the best LDA models.

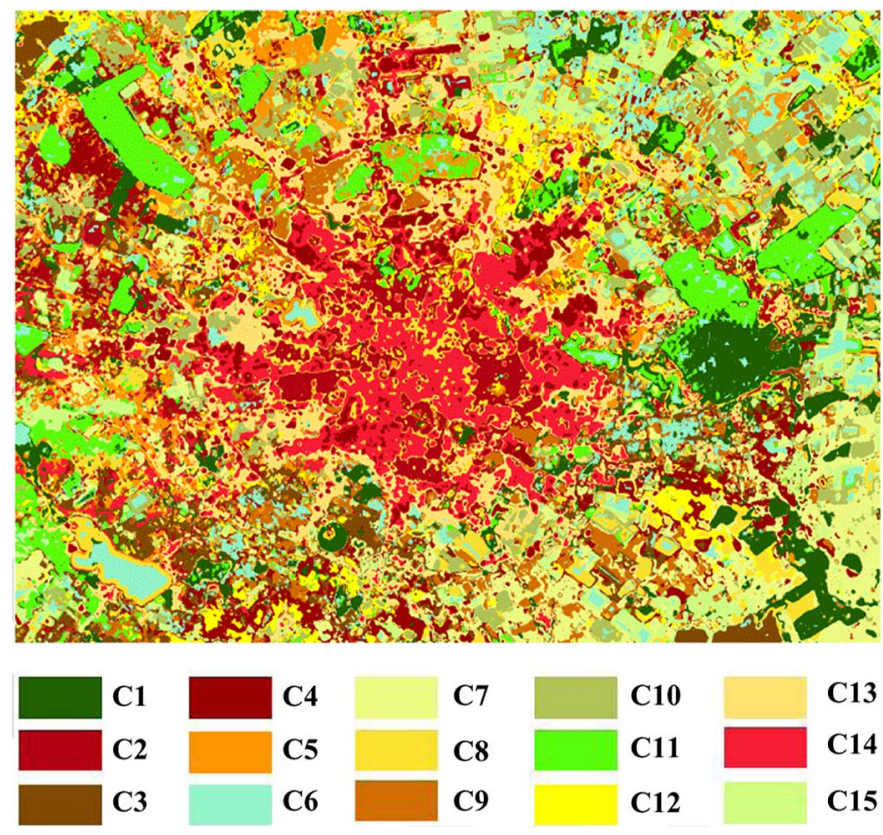

Fig. 6. Classification of the Earth's surface dynamic evolution. The legend specifies the classes' indexes.

The selection of the dictionary and the optimum document size were determined by comparing the values of perplexity according to Fig. 5. According to this assessment, it is found that the LDA model defined using a dictionary of 200 words and a document size of $6 \times 6$ pixels is characterized by the lowest value of the perplexity. Regarding the number of topics, we can observe a local minimum and, thus, an optimum value for a number of 15 topics.

\section{B. Experimental Results}

Considering the setup process described in Section V-A, we resume here the selected parameters for the proposed latent analysis of Earth's surface dynamic evolution:

1) size of the sliding window for change detection: $10 \times 10$ pixels;

2) size of the dictionary for the LDA model: 200 words;

3) size of the document for the LDA model: $6 \times 6$ pixels;

4) number of topics for the LDA analysis: 15 .

There are several processing steps, involving the analysis of a SITS of 11 scenes, from which we extract a set of 40 different change measures. In terms of time consumptions, we mention 
that, on a desktop personal computer with a 2.4-GHz quad-core CPU and 16 GB of RAM, each run of $k$-means took about $2 \mathrm{~h}$ and each run of LDA training process required about $1.5 \mathrm{~h}$. The classification (topic estimation) step of LDA is faster, requiring about 15 min.

The obtained results (Fig. 6) are presented as a land cover classification, each class (LDA topic) portraying areas with a similar evolution (concerning the type and the time of changes that occurred) during the entire SITS acquisition period. The Earth's surface is characterized based on the dynamic evolution of objects and structures on the ground in the analyzed period. Without previous information explaining certain transformations, a specific description of the retrieved LDA topics is complicated, such that the legend of the obtained map specifies only the indexes for the identified classes.

The specific features of each class (LDA topic) can be described in terms of temporal signatures according to the dynamic evolution of the pixels included in that class. One temporal signature, subsequent to one pixel, is given by all the values in the CMTS corresponding to the coordinates of that pixel. Each temporal signature is characterized by four different components, measured with the four similarity measures. The components defining the dynamic evolutions for all the classes in Fig. 6 are shown in Fig. 7(a) (for the CC-based evolution), Fig. 7(b) (for the KLD-based evolution), Fig. 7(c) (for the CIbased evolution), and Fig. 7(d) (for the NCD-based evolution). We mention that we used the same colors for the classification as well as for the dynamic evolution representation. On the $x$ axis, we can find the image pairs (see Table II) for which the degree of change on the $y$-axis was computed. The obtained values were normalized between zero and one, such that all the identified transformations have the same influence during the LDA modeling.

We observe that the radiometric changes were smaller than the texture and spectral ones. This can be explained by the fact that the time interval for the analyzed SITS is included in one year, which is too short for a city as large as Bucharest to suffer important transformations. All the classes have a similar evolution until the end of July, when some of the regions, corresponding to five classes, continue to transform while the rest of them tend to remain unchanged.

The spectral evolution is characterized by a higher degree of change, from 0.7 to 0.93 . The dynamic evolution computed based on the KLD is similar for all the classes until July 3 when all begin to be defined by a different variation. The dynamic evolution computed based on the CI is more uniform for all the classes, with small differences between the image pairs.

The specific of texture changes consists of dividing this dynamic evolution in two groups of classes. The first has a higher and constant degree of change. The second group is characterized by smaller values and a marked variation.

The presented approach is innovative in the field of SITS modeling, and the related ground truth is more difficult to obtain. Precise knowledge about the exploration areas is required. A comparison with a human-based already existing classification can be very helpful for the interpretation of certain areas. Table III presents a comparison between the obtained results (Fig. 6) and the Corinne Land Cover (CLC) map (Fig. 8),

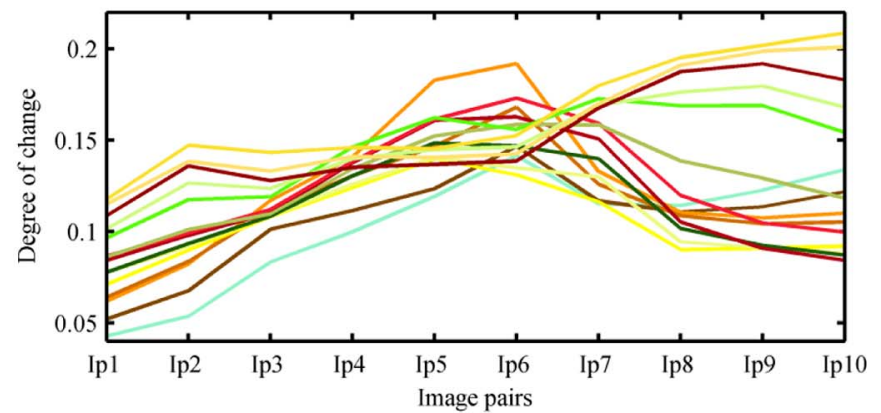

(a)

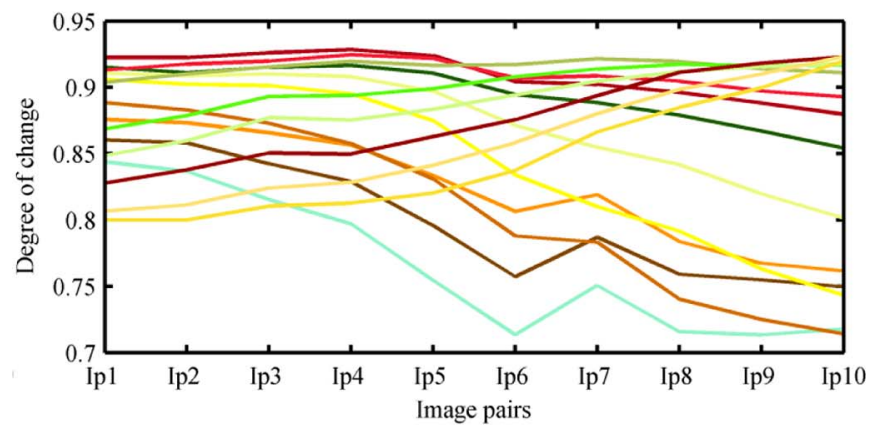

(b)

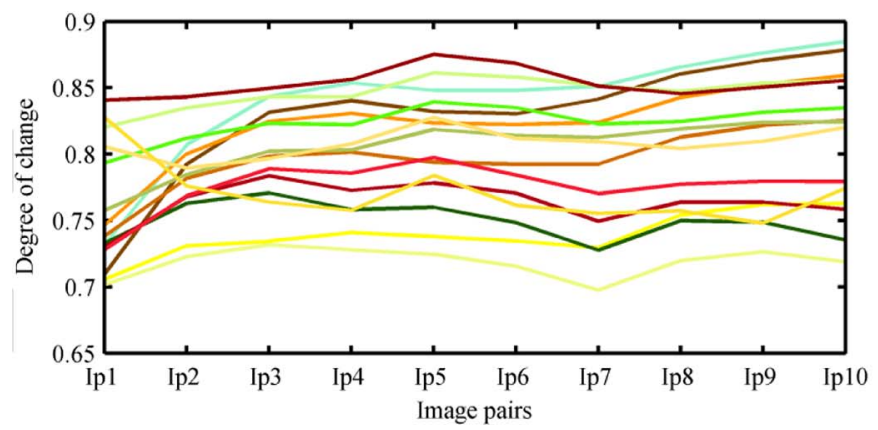

(c)

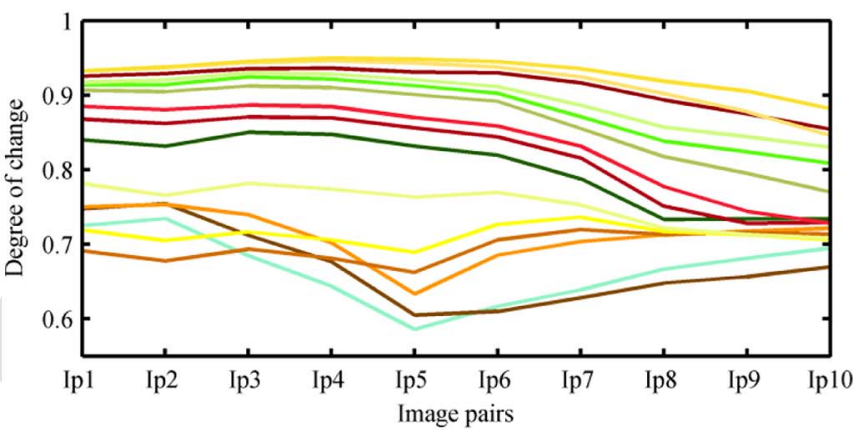

(d)

Fig. 7. Dynamic evolution corresponding to each of the 15 classes in Fig. 6 computed based on the following similarity measures: (a) CC, (b) KLD, (c) CI, and (d) NCD.

offering information about how much the LDA and CLC classes fit. The table provides the number of pixels that have identical labels. An increase in the number of pixels ensures a higher degree of similarity between the LDA and CLC classes.

It is important to mention that the two classifications present the Earth surface from different perspectives. The Corinne classification divides the analyzed area in four types of static areas according to a general land use: urban area, forest, water, and agriculture. The method proposed in this paper offers a more 
TABLE III

COMPARISON BETWEEN LDA CLASSIFICATION AND CLC MAP

\begin{tabular}{|c|c|c|c|c|}
\hline $\begin{array}{c}\text { CLC } \\
\text { class }\end{array}$ & $\begin{array}{c}\text { Urban } \\
\text { area }\end{array}$ & Forest & Water & Agriculture \\
\hline $\begin{array}{c}\text { LDA } \\
\text { class }\end{array}$ & & & & \\
\hline 1 & 5515 & 48866 & 5489 & 68099 \\
\hline 2 & 58734 & 2455 & 1327 & 51068 \\
\hline 3 & 7597 & 1972 & 839 & 75332 \\
\hline 4 & 34806 & 1316 & 236 & 75597 \\
\hline 5 & 16448 & 5600 & 1850 & 61438 \\
\hline 6 & 3468 & 9450 & 8892 & 79927 \\
\hline 7 & 6607 & 1398 & 871 & 100420 \\
\hline 8 & 28913 & 2940 & 6302 & 52656 \\
\hline 9 & 9594 & 839 & 908 & 76125 \\
\hline 10 & 3573 & 530 & 382 & 93624 \\
\hline 11 & 3919 & 64570 & 4811 & 44885 \\
\hline 12 & 13007 & 2824 & 3300 & 68881 \\
\hline 13 & 72956 & 843 & 810 & 89956 \\
\hline 14 & 105100 & 446 & 652 & 30919 \\
\hline 15 & 10198 & 1063 & 1043 & 89036 \\
\hline
\end{tabular}

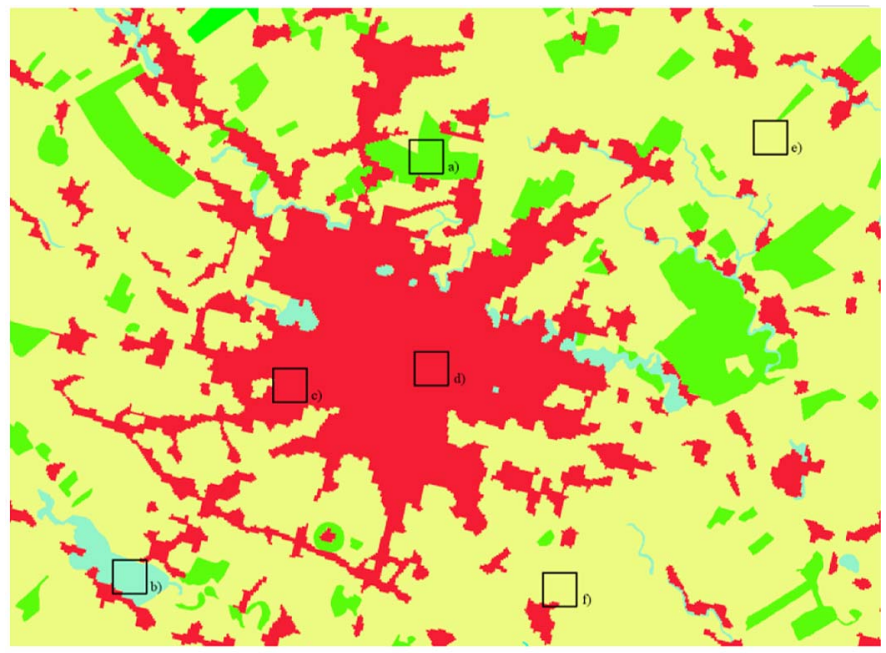

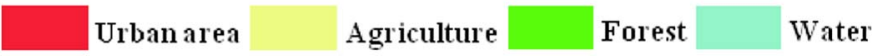

Fig. 8. CLC map for the analyzed area (Bucharest and surroundings, Romania).

generous distribution of land cover classes based on the regions' dynamic evolution. As we can see in the following figures, one static CLC class can be split into several dynamic LDA classes. We have identified several urban classes, forest types, and agricultural fields. Our method is able to distinguish between several residential areas inside the city, due to the variation of the vegetation component encapsulated in the resolution cell (Figs. 11 and 12). It can also separate different agricultural classes according to the vegetation evolution, the type of crop,
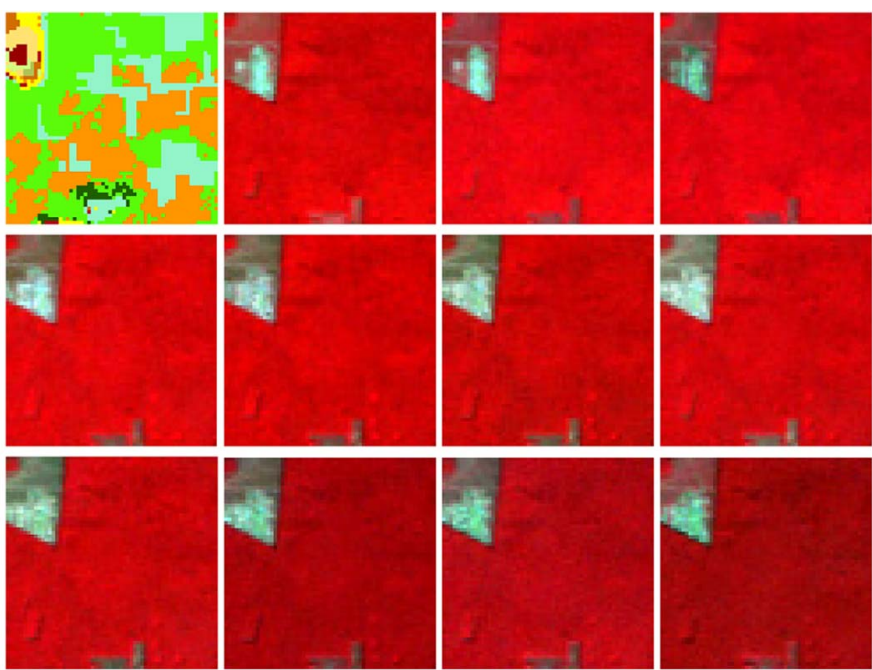

Fig. 9. Area a) in Fig. 8. The first tile represents the result of our classification for area a); the rest of the tiles are extracted from the initial SITS.

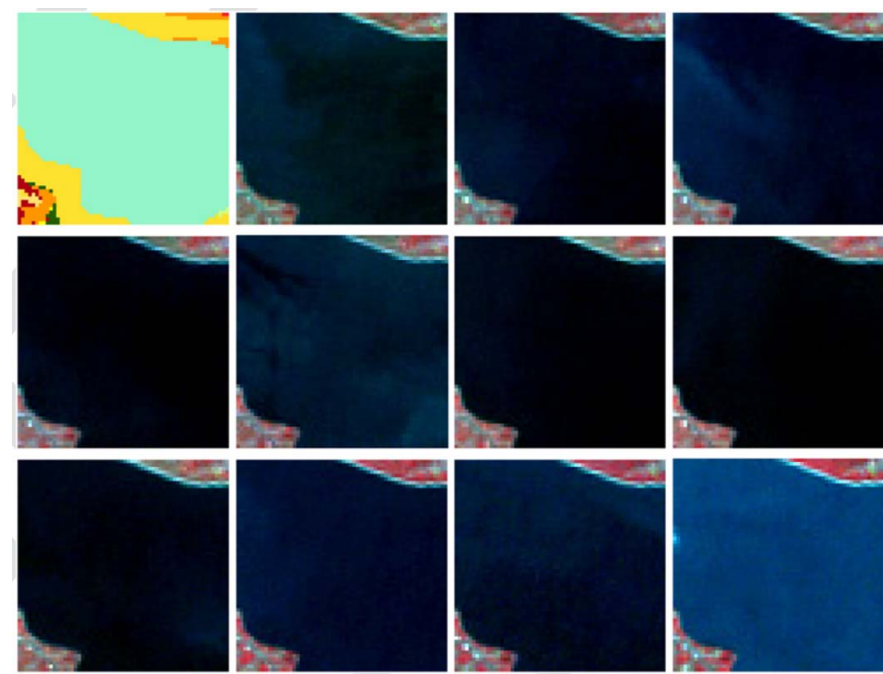

Fig. 10. Area b) in Fig. 8. The first tile represents the result of our classification for area $b$ ); the rest of the tiles are extracted from the initial SITS.
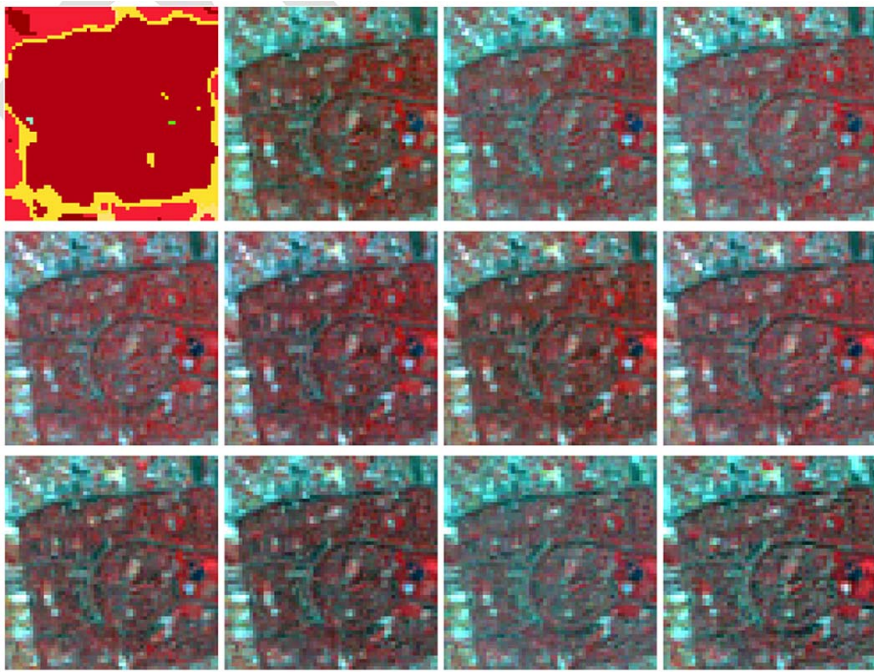

Fig. 11. Area c) in Fig. 8. The first tile represents the result of our classification for area c); the rest of the tiles are extracted from the initial SITS. 


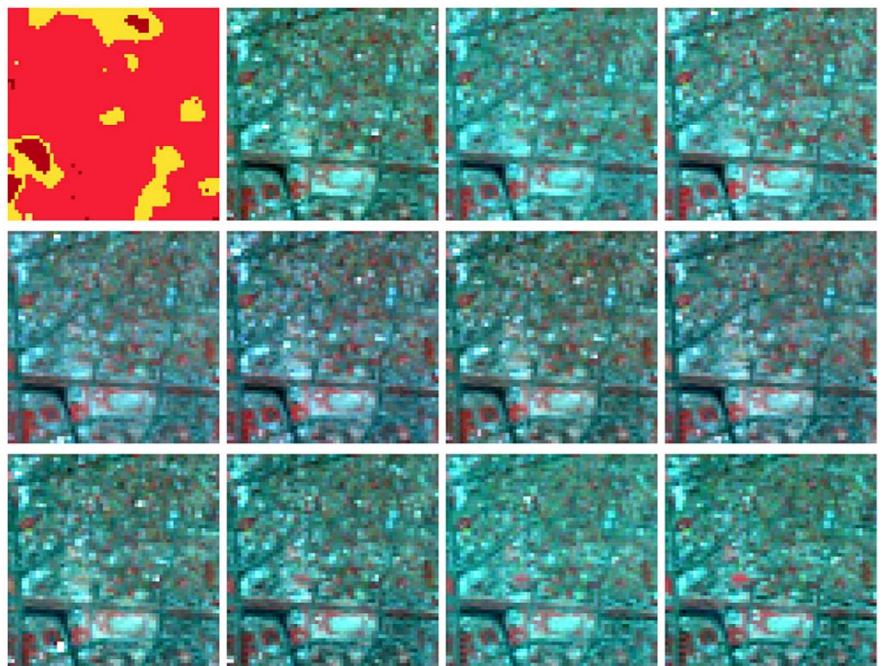

Fig. 12. Area d) in Fig. 8. The first tile represents the result of our classification for area $\mathrm{d}$ ); the rest of the tiles are extracted from the initial SITS.
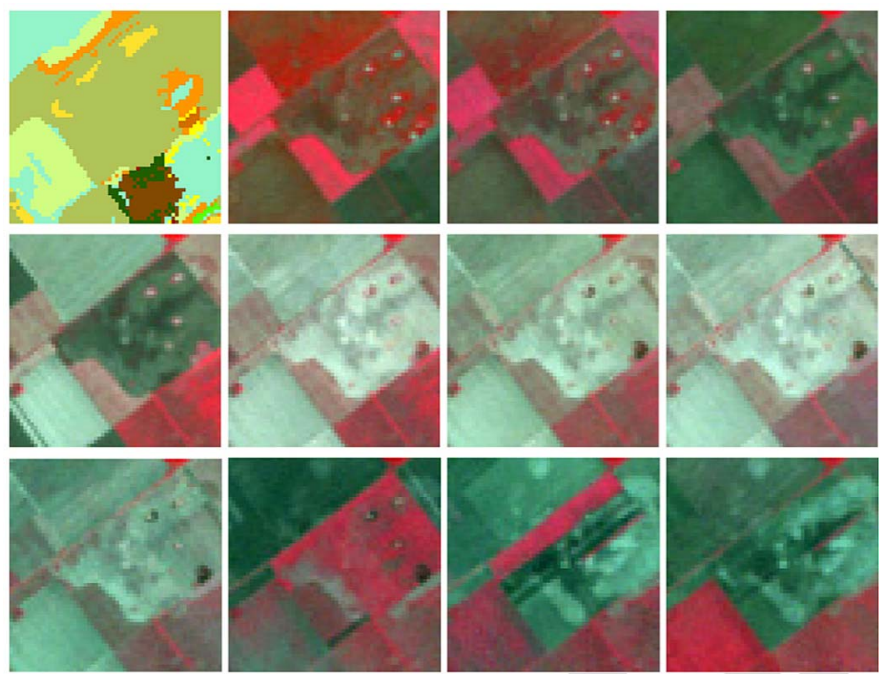

Fig. 13. Area e) in Fig. 8. The first tile represents the result of our classification for area e); the rest of the tiles are extracted from the initial SITS.
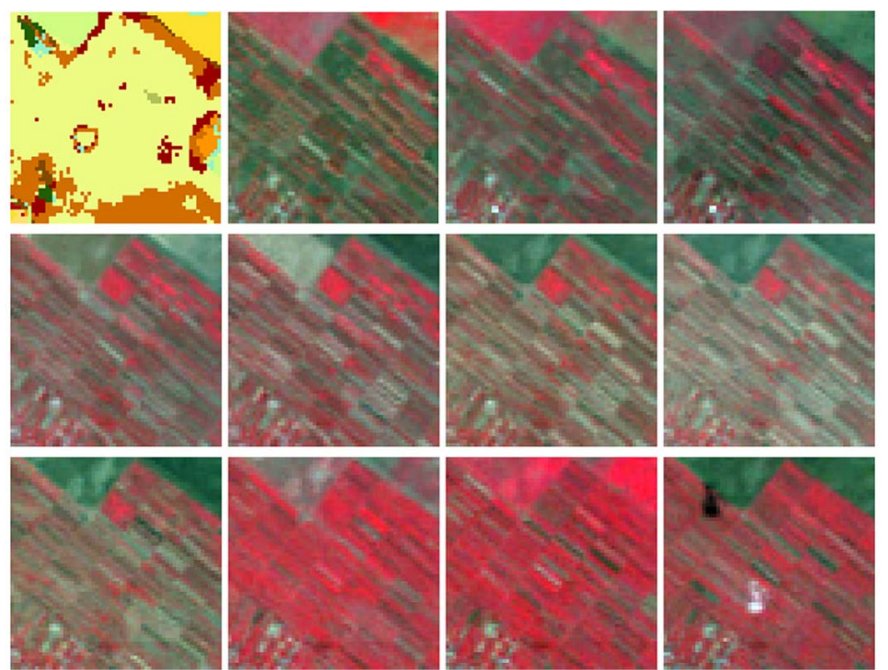

Fig. 14. Area f) in Fig. 8. The first tile represents the result of our classification for area f); the rest of the tiles are extracted from the initial SITS. the soil processing, and the granularity of parceling (Figs. 13 and 14), as well as forested areas based on the tree types.

In order to exemplify our statements, we take out six tiles from the analyzed scene, comparing the CLC map with our results, and present the corresponding region in all the images of the initial SITS. The positioning of the tiles in the scene is shown in Fig. 8. The corresponding LDA classification and the patches extracted from the SITS are shown in Figs. 9-14.

The first analyzed area, i.e., area a), was labeled in the CLC map mostly as a forest area. The method described in this paper was able to separate four classes of the forest dynamic evolution. The classification corresponding to this area is given in the first image in Fig. 9, while the rest of the images illustrate the same area within the initial SITS.

The second area, i.e., area $b$ ), is included in the CLC water class. Our results in Fig. 10 treat it as a single class, too. However, another specific property of the proposed method consists of grouping in the same class areas with different physical interpretations but with the same type of dynamic evolution, such as water and out-of-crop regions (blue class in Fig. 6).

The next two areas, i.e., areas $c$ ) and $d$ ), were both labeled as an urban area in the CLC map. The proposed method retrieves regions with distinct features inside (Figs. 11 and 12). This is due to the vegetation in the city, the type of buildings, and the distance between them. All the structures integrated in a resolution cell affect the dynamic of the land cover. In Fig. 16, we see two very high resolution (VHR) images representing the areas $c$ ) and $d$ ), where we can actually observe regions containing different types of buildings.

The last two analyzed areas, i.e., areas e) and f), present agricultural areas according to the CLC map. The proposed method differentiates between a wide range of dynamic evolution classes inside these areas based on the type of crop, the parceling characteristics, or the harvesting period (Figs. 13 and 14). Even if we cannot assign a particular knowledge-based label concerning the type of crop evolution due to the private nature of the information, we are however able to identify the bare land annotated as class 15 in the resulted map (Fig. 6).

Moreover, the results shown in Fig. 13 illustrate the latent character of the methodology introduced in this paper. Using the change information computed with the four similarity measures, the LDA model was able to retrieve similar areas that a user with no specific knowledge background cannot find. We can observe in this tile that the blue area (class 6) represents agriculture. However, we can also view in Fig. 6 that this class is also assigned to lakes and rivers. The explanation is given by several studies regarding the nature of the soil covering the south southeast side of Romanian territory, including the scene analyzed in this paper. It is stated in [23] that Bucharest is surrounded by a specific form of relief called microdepression [24]. Its main characteristic refers to the fact that it is impermeable and has the shape of a funnel. Therefore, it retains water for a prolonged period of time until that drains or evaporates.

The LDA classification is supported in this direction by the weather forecast for the period when the SITS was acquired, i.e., May-September 2007 (Table IV). This information was 
TABLE IV

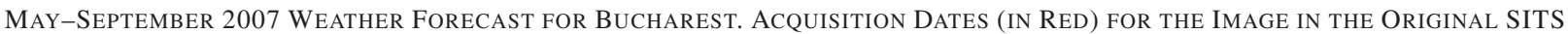

\begin{tabular}{|c|c|c|c|c|c|c|c|c|c|c|c|c|c|c|c|c|}
\hline MAY & 1 & 2 & 3 & 4 & 5 & 6 & 7 & 8 & 9 & 10 & 11 & 12 & 13 & 14 & 15 & 16 \\
\hline rainfall & 0 & 0 & 0 & 0 & 0 & 0 & 1.3 & 0 & 0 & 0 & 0 & 0 & 0 & 0 & 0 & 0 \\
\hline Weather forecast & & $\begin{array}{l}\text { Rain/ } \\
\text { drizzle }\end{array}$ & & $\begin{array}{l}\text { Rain/ } \\
\text { drizzle }\end{array}$ & & $\begin{array}{l}\text { Rain/ } \\
\text { drizzle }\end{array}$ & $\begin{array}{c}\text { Rain / } \\
\text { drizzle } \\
\text { Lightning }\end{array}$ & $\begin{array}{l}\text { Rain/ } \\
\text { drizzle }\end{array}$ & & & & & & & & \\
\hline $\begin{array}{l}\text { image acquisition } \\
\text { date }\end{array}$ & & & & & & & & & & & & & & & & no clouds \\
\hline
\end{tabular}

\begin{tabular}{|c|c|c|c|c|c|c|c|c|c|c|c|c|c|c|c|}
\hline \multirow{2}{*}{$\begin{array}{l}\mathrm{MAY} \\
\text { rainfall } \\
\end{array}$} & 17 & 18 & 19 & 20 & 21 & 22 & 23 & 24 & 25 & 26 & 27 & 28 & 29 & 30 & 31 \\
\hline & 0 & 0 & 15.9 & 1.8 & 1 & 0 & 0.5 & 3.1 & 0 & 0 & 0 & 10.5 & 5.4 & 1 & 0 \\
\hline Weather forecast & & $\begin{array}{c}\text { Rain / } \\
\text { drizzle } \\
\text { Lightning }\end{array}$ & $\begin{array}{l}\text { Rain/ } \\
\text { drizzle }\end{array}$ & $\begin{array}{l}\text { Rain/ } \\
\text { drizzle }\end{array}$ & Lightning & $\begin{array}{l}\text { Rain / } \\
\text { drizzle }\end{array}$ & $\begin{array}{c}\text { Rain/ } \\
\text { drizzle } \\
\text { Lightning }\end{array}$ & Lightning & Lightning & & $\begin{array}{c}\text { Rain / } \\
\text { drizzle } \\
\text { Lightning }\end{array}$ & $\begin{array}{c}\text { Rain/ } \\
\text { drizzle } \\
\text { Lightning }\end{array}$ & $\begin{array}{l}\text { Rain/ } \\
\text { drizzle }\end{array}$ & $\begin{array}{l}\text { Rain/ } \\
\text { drizzle }\end{array}$ & $\begin{array}{c}\text { Rain / drizzle } \\
\text { Hailstones } \\
\text { Lightning }\end{array}$ \\
\hline $\begin{array}{l}\text { image acquisition } \\
\text { date }\end{array}$ & & & & & & & & & no clouds & & & & & & \\
\hline
\end{tabular}

\begin{tabular}{|c|c|c|c|c|c|c|c|c|c|c|c|c|c|c|c|c|}
\hline JUNE & 1 & 2 & 3 & 4 & 5 & 6 & 7 & 8 & 9 & 10 & 11 & 12 & 13 & 14 & 15 & \\
\hline rainfall & 4.1 & 0 & 0 & 0 & 0 & 0 & 0 & 0 & 0 & 15.1 & 0 & 0 & 0 & 0 & 1.5 & \\
\hline $\begin{array}{c}\text { Weather forecast } \\
\text { Rain/ } \\
\text { drizzle } \\
\text { Lightning }\end{array}$ & & & $\begin{array}{c}\text { Rain/ } \\
\text { drizle }\end{array}$ & & & & & $\begin{array}{c}\text { Rain/drizle } \\
\text { Hailstones } \\
\text { Lightning }\end{array}$ & & & & & $\begin{array}{c}\text { Rain/ } \\
\text { dirzzle } \\
\text { Lightning }\end{array}$ & $\begin{array}{c}\text { Rain/drizzle } \\
\text { Lightning }\end{array}$ & \\
\hline $\begin{array}{c}\text { image acquisition } \\
\text { date }\end{array}$ & & & & & & & & & & no clouds & & & & & & \\
\hline
\end{tabular}

\begin{tabular}{|c|c|c|c|c|c|c|c|c|c|c|c|c|c|c|c|}
\hline \multirow{2}{*}{$\begin{array}{c}\text { JUNE } \\
\text { rainfall }\end{array}$} & 16 & 17 & 18 & 19 & 20 & 21 & 22 & 23 & 24 & 25 & 26 & 27 & 28 & 29 & 30 \\
\hline & 0.3 & 0 & 0 & 0 & 0 & 1.3 & 0.8 & 0 & 0 & 0 & 0 & 0.5 & 0 & 4.9 & 0 \\
\hline Weather forecast & Lightning & $\begin{array}{l}\text { Rain/ } \\
\text { drizzle }\end{array}$ & & $\begin{array}{l}\text { Rain/ } \\
\text { drizzle }\end{array}$ & $\begin{array}{c}\text { Rain/ } \\
\text { drizzle } \\
\text { Lightning }\end{array}$ & & $\begin{array}{l}\text { Rain/ } \\
\text { drizzle }\end{array}$ & & & & $\begin{array}{c}\text { Rain / } \\
\text { drizzle } \\
\text { Lightning }\end{array}$ & & $\begin{array}{c}\text { Rain / } \\
\text { drizzle } \\
\text { Lightning }\end{array}$ & & Rain / drizzle \\
\hline $\begin{array}{c}\text { image acquisition } \\
\text { date }\end{array}$ & & & & & & & & & & & no clouds & & & & \\
\hline
\end{tabular}

\begin{tabular}{|c|c|c|c|c|c|c|c|c|c|c|c|c|c|c|c|c|}
\hline JULY & 1 & 2 & 3 & 4 & 5 & 6 & 7 & 8 & 9 & 10 & 11 & 12 & 13 & 14 & 15 & 16 \\
\hline rainfall & 0 & 0 & 0 & 0 & 0 & 0 & 0 & 0 & 0 & 0 & 0.5 & 21.8 & 0 & 0 & 0 & 0 \\
\hline \begin{tabular}{c} 
Weather forecast \\
\hline $\begin{array}{c}\text { image acquisition } \\
\text { date }\end{array}$
\end{tabular} & & & & & & & & & $\begin{array}{c}\text { Rain/ } \\
\text { drizzle } \\
\text { Lightning }\end{array}$ & $\begin{array}{c}\text { Rain/ } \\
\text { drizle } \\
\text { Lightning }\end{array}$ & $\begin{array}{c}\text { Rain/ } \\
\text { drizzle } \\
\text { Lightning }\end{array}$ & & & \\
\hline
\end{tabular}

\begin{tabular}{|c|c|c|c|c|c|c|c|c|c|c|c|c|c|c|c|c|}
\hline JULY & 17 & 18 & 19 & 20 & 21 & 22 & 23 & 24 & 25 & 26 & 27 & 28 & 29 & 30 & 31 & \\
\hline rainfall & 0 & 0 & 0 & 0 & 0 & 0 & 0 & 0 & 0 & 0 & 0 & 0 & 0 & 0 & 0 & \\
\hline \begin{tabular}{c} 
Weather forecast \\
\hline $\begin{array}{c}\text { image acquisition } \\
\text { date }\end{array}$
\end{tabular} & & & & & & & & & & & & & & Rain/drizzle & \\
\hline
\end{tabular}

\begin{tabular}{|c|c|c|c|c|c|c|c|c|c|c|c|c|c|c|c|c|}
\hline AUGUST & 1 & 2 & 3 & 4 & 5 & 6 & 7 & 8 & 9 & 10 & 11 & 12 & 13 & 14 & 15 & 16 \\
\hline rainfall & 2.8 & 10.5 & 0 & 0 & 0 & 0 & 27.4 & 18.2 & 0 & 0 & 0 & 0 & 0 & 0 & 0 & 0 \\
\hline Weather forecast & $\begin{array}{c}\text { Rain/ } \\
\text { drizzle } \\
\text { Lightning }\end{array}$ & & & & $\begin{array}{l}\text { Rain/ } \\
\text { drizzle }\end{array}$ & $\begin{array}{c}\text { Rain/ } \\
\text { drizzle } \\
\text { Lightning }\end{array}$ & $\begin{array}{c}\text { Rain/ } \\
\text { drizzle } \\
\text { Lightning }\end{array}$ & $\begin{array}{l}\text { Rain/ } \\
\text { drizzle }\end{array}$ & & & & & & & & \\
\hline $\begin{array}{l}\text { image acquisition } \\
\text { date }\end{array}$ & & & & no clouds & & & & & & & & & & & & \\
\hline
\end{tabular}

\begin{tabular}{|c|c|c|c|c|c|c|c|c|c|c|c|c|c|c|c|c|}
\hline AUGUST & 17 & 18 & 19 & 20 & 21 & 22 & 23 & 24 & 25 & 26 & 27 & 28 & 29 & 30 & 31 & \\
\hline rainfall & 0 & 0 & 0 & 0.3 & 0 & 0 & 0 & 0 & 0 & 0 & 28.5 & 0 & 0 & 0 & 0.3 & \\
\hline Weather forecast & & & $\begin{array}{c}\text { Rain/ } \\
\text { drizzle } \\
\text { Lightning }\end{array}$ & & & & & & & $\begin{array}{c}\text { Rain / } \\
\text { drizle } \\
\text { Lightning }\end{array}$ & $\begin{array}{l}\text { Rain/ } \\
\text { drizzle }\end{array}$ & & & $\begin{array}{c}\text { Rain/ } \\
\text { drizle } \\
\text { Lightning }\end{array}$ & Lightning & \\
\hline $\begin{array}{c}\text { image acquisition } \\
\text { date }\end{array}$ & & & & no clouds & & & & & & & & & no clouds & & & \\
\hline
\end{tabular}

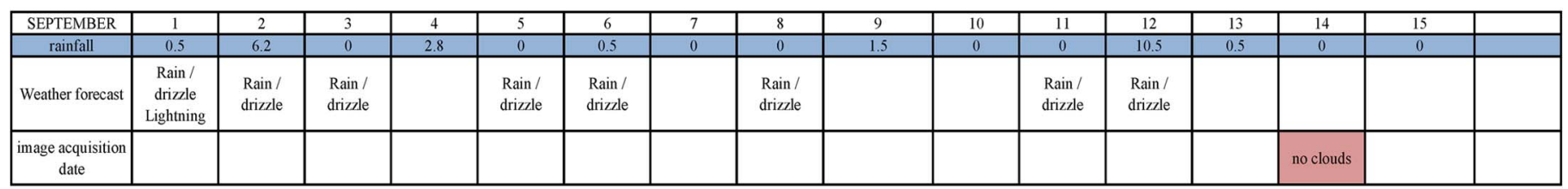

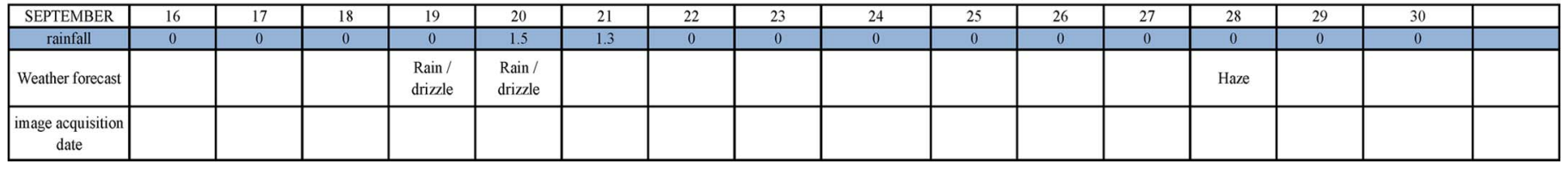



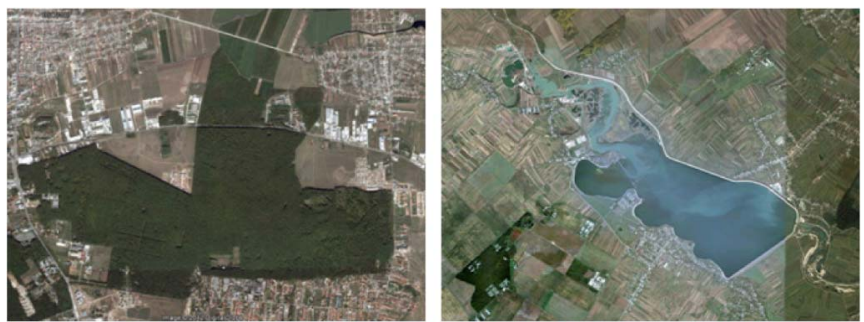

Fig. 15. VHR images illustrating (left) area $a$ ) and (right) area $b$ ) with surroundings.
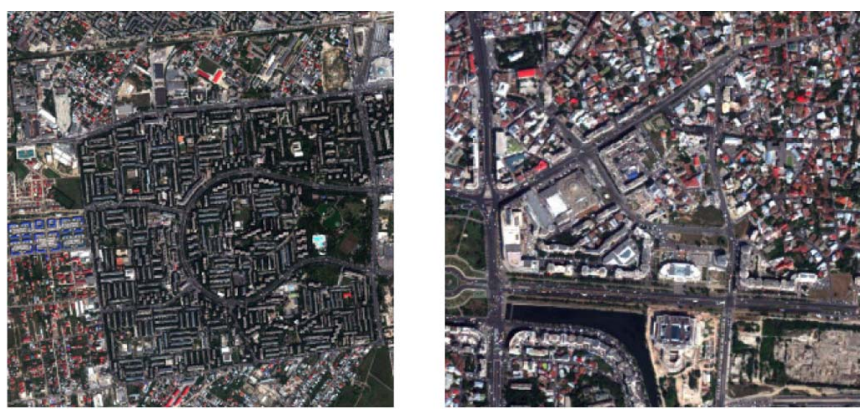

Fig. 16. VHR images [21] illustrating (left) area $c$ ) and (right) area $d$ ) with surroundings.
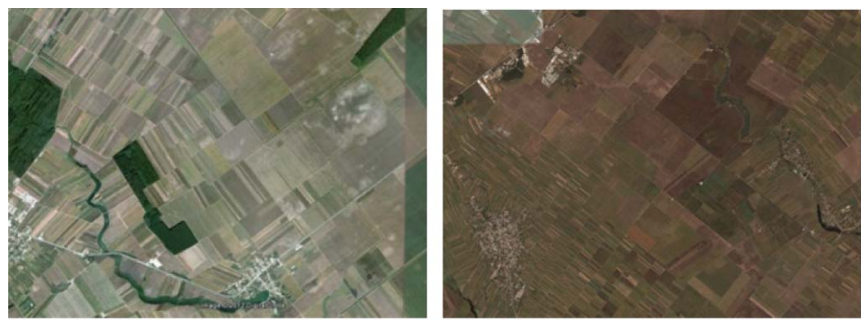

Fig. 17. VHR images illustrating (left) area $e$ ) and (right) area $f$ ) with surroundings.

extracted from weather archives [25]. We should consider that this information is just a forecast and not a fact, explaining, in the presence of microdepressions, the discrepancy between the acquisition date and the rain periods. There are also paper news from the summer of 2007 describing high temperatures and also heavy rain alerts [26].

In the absence of precise ground truth, we can use a series of VHR images in order to support a better visual inspection of the considered region. Images in Figs. 15 and 17 were extracted from Google Earth and were acquired in 2007, the time of the analyzed SITS.

The tiles shown in Figs. 15-17 help sustain the variety of classes extracted using the proposed algorithm. As can be noticed, an increase in resolution allows us to observe a larger variety of structures that can strongly influence the land cover dynamics.

\section{CONCLUSION}

The method described by the authors has offered a new approach for SITS analysis based on the dynamic evolution of the Earth's surface which comes to complement the temporal information extracted by previously known algorithms. We applied four algorithms of change detection using four different similarity measures that capture the differences between pairs of consecutive acquisitions to extract new features for describing the evolution of scene dynamics. The complementarity of the CC, the KLD, the CI, and the NCD is ensured by the information derived from the basic data characteristics (radiometry, spectral, and texture).

The idea behind the proposed approach consists of the conversion of the original Landsat SITS in a new image time series. After computing the differences between consecutive images in the original data set using all four similarity measures, the resulting change maps were gathered in a new image stack called the CMTS. Therefore, a pixel is no longer characterized by the static information of the original scene but expresses the dynamics of the structures enclosed in the resolution cell. This evolution can be observed using temporal signatures such as those in Fig. 7.

The CMTS was analyzed by applying a latent discovery method scene classification initially developed for text analysis-LDA. Several text-image analogies have been used. The LDA model has the ability to group words located at distant positions in the feature space, as well as to extract classes that have high semantic meaning. However, it is important to note that the LDA model cannot be entirely viewed as a clustering or classification method, as much as an algorithm for discovering heterogeneous groupings enclosing semantic meaning. The method has the advantage of good performances even for small amounts of training data with no precise information about the classes to be obtained. The main characteristic refers to its ability of retrieving latent information that a user is not able to find easily.

The purpose of the presented case study was to analyze the evolution of the Earth surface by the changes that occurred, considering the influence of seasons on vegetation, the crop lifetime, and human activities. The LDA modeling is an efficient method to build a figurative classification of the scene dynamic evolution.

The results were compared to the CLC classification. The main difference is that, while CLC defines a number of four static classes, the proposed algorithm is able to identify a wide range of classes with dynamic evolution. Our method can distinguish between several urban areas and types of forests and can also separate different agricultural classes according to the vegetation evolution, types of crop, soil processing, granularity of parceling, and harvesting period. The LDA model revealed the latent information encapsulated in the scene, similarly labeling the microdepressions filled with water by heavy rains and the lakes or rivers. They are all characterized by alike evolutions, as they contain water.

A major drawback of this method is the lack of reliable ground truth related to the dynamic evolution of the land cover. For this reason, the validation may often be based on VHR images outside the database, acquired in the same time interval.

The competence of the proposed methodology to retrieve latent information from a low-resolution Landsat image time series has been demonstrated. It can be applied also for medium SITS, but it is not extendable for high-resolution and VHR 
SITSs. The change detection algorithms require the images to be very well superposed in order to obtain fair relevant results. When increasing the spatial resolution, a multitude of details become available, and the coregistration is extremely difficult. Moreover, the high-resolution and VHR sensors usually acquire images with different incidence angles and at a different moment of the day every time they survey an area. Given this matter, the shape of objects and that of their shadows can have significant variations, affecting the change detection process. It is thus very unlikely to obtain a perfectly coregistered VHR SITS, such that the method proposed in this paper can be applied for its analysis. It is possible, however, to obtain an image time series that fulfills these requirements, but the process involves great expenses to be considered.

\section{ACKNOWLEDGMENT}

The authors would like to thank Prof. A. Badea for his helpful pieces of advice regarding result understanding, interpretation, and validation.

\section{REFERENCES}

[1] S. Laxman and P. S. Sastry, "A survey of temporal data mining," Sadhana, vol. 31, pt. 2, pp. 173-198, Apr. 2006.

[2] L. Gueguen and M. Datcu, "Image time-series data mining based on the information-bottleneck principle," IEEE Trans. Geosci. Remote Sens., vol. 45, no. 4, pp. 827-838, Apr. 2007.

[3] A. Julea, N. Méger, P. Bolon, C. Rigotti, M. P. Doin, C. Lasserre, E. Trouvé, and V. N. Lăzărescu, "Unsupervised spatiotemporal mining of satellite image time series using grouped frequent sequential patterns," IEEE Trans. Geosci. Remote Sens., vol. 49, no. 4, pp. 1417-1430, Apr. 2011

[4] P. Heas and M. Datcu, "Modeling trajectory of dynamic clusters in image time-series for spatio-temporal reasoning," IEEE Trans. Geosci. Remote Sens., vol. 43, no. 7, pp. 1635-1647, Jul. 2005.

[5] L. Gueguen and M. Datcu, "Mixed information measure: Application to change detection in Earth observation," presented at the 5th Int. Workshop Analysis Multi-temporal Remote Sensing Images, Groton, CT, Jul. 28-30, 2009.

[6] E. P. Crist and R. C. Cicone, "A physically-based transformation of thematic mapper data-The TM tasselled cap," IEEE Trans. Geosci. Remote Sens., vol. GE-22, no. 3, pp. 256-263, May 1984.

[7] J. Inglada and A. Giros, "On the possibility of automatic multisensor image registration," IEEE Trans. Geosci. Remote Sens., vol. 42, no. 10, pp. 2104-2120, Oct. 2004.

[8] D. Faur, C. Vaduva, I. Gavat, and M. Datcu, "An information theory based image processing chain for change detection in Earth observation," in Proc. IWSSIP, Bratislava, Slovak Republik, Jun. 25-28, 2008, vol. 15, pp. 129-132.

[9] L. Alparone, B. Aiazzi, S. Baronti, and A. Garzelli, "An informationtheoretic feature for multi-temporal analysis of SAR images," in Proc. ESA-EUSC, Torrejon, Madrid, Spain, Nov. 2006.

[10] M. Li, X. Chen, Li, B. Ma, and P. M. B. Vitanyi, "The similarity metric," IEEE Trans. Inf. Theory, vol. 50, no. 12, pp. 3250-3264, Dec. 2004.

[11] C. Vaduva, D. Faur, A. A. Popescu, I. Gavat, and M. Datcu, "Semantic map generation from satellite images for humanitarian scenarios applications," in Proc. Adv. Concepts Intell. Vis. Syst. Conf., Juan-les-Pins, France, Oct. 20-24, 2008, pp. 808-817.

[12] D. M. Blei, A. Y. Ng, and M. I. Jordan, "Latent Dirichlet allocation," J. Mach. Learn. Res., vol. 3, pp. 993-1022, Mar. 2003.

[13] K. Barnard, P. Duygulu, D. Forsyth, N. de Freitas, D. M. Blei, and M. Jordan, "Matching words and pictures," J. Mach. Learn. Res., vol. 3, pp. 1107-1135, Mar. 2003.

[14] S. Kullback and R. Leibler, "On information and sufficiency," Ann. Math. Stat., vol. 22, no. 1, pp. 79-86, Mar. 1951.

[15] M. Lienou, H. Maitre, and M. Datcu, "Semantic annotation of satellite images using latent Dirichlet allocation," IEEE Geosci. Remote Sens. Lett., vol. 7, no. 1, pp. 28-32, Jan. 2010.
[16] D. Bratasanu, I. Nedelcu, and M. Datcu, "Bridging the semantic gap for satellite image annotation and automatic mapping applications," IEEE J. Sel. Topics Appl. Earth Observ. Remote Sens., vol. 4, no. 1, pp. 193-204, Mar. 2010.

[17] G. Chander, B. L. Markham, and D. L. Helder, "Summary of current radiometric calibration coefficients for Landsat MSS, TM, ETM +, and EO-1 ALI sensors," Remote Sens. Environ., vol. 113, no. 5, pp. 893-903, May 15, 2009.

[18] M. J. Canty, A. A. Nielsen, and M. Schmidt, "Automatic radiometric normalization of multitemporal satellite imagery," Remote Sens. Environ., vol. 91, no. 3/4, pp. 441-451, Jun. 30, 2004.

[19] L. Gueguen and M. Datcu, "A similarity metric for retrieval of compressed objects: Application for mining satellite image time series," IEEE Trans. Knowl. Data Eng., vol. 20, no. 4, pp. 562-575, Apr. 2008.

[20] [Online]. Available: http://earthexplorer.usgs.gov/

[21] [Online]. Available: www.digitalglobe.com

[22] P. S. Chavez, Jr., "Image-based atmospheric corrections-Revisited and improved," Photogramm. Eng. Remote Sens., vol. 62, no. 9, pp. 10251036, Sep. 1996.

[23] A. Badea, "Studiul efectelor impactului lucrarilor de imbunatatiri funciare asupra conditiilor fizico-geografice din Baraganul de Sud utilizand mijloace informatice de colectare, analiza si gestionare a datelor," Ph.D. dissertation, Faculty Geography, Univ. Bucharest, Bucureşti, Romania, 2004.

[24] A. Gherghina, F. Grecu, and P. Molin, "Morphometrical analysis of microdepressions in the central Baragan plain (Romania)," Revista de geomorfologie, vol. 10, pp. 31-38, 2008.

[25] [Online]. Available: www.freemeteo.com

[26] [Online]. Available: http://vremea.meteoromania.ro/taxonomy/term/206

[27] F. Petitjean, J. Inglada, and P. Garcarski, "Satellite image time series analysis under time warping," IEEE Trans. Geosci. Remote Sens., vol. 50, no. 8, pp. 3081-3095, Aug. 2012.

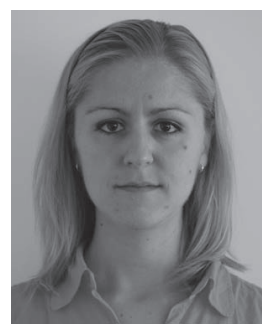

Corina Văduva received the B.S. and Ph.D. degrees in electronics and telecommunications from the University "Politehnica" of Bucharest (UPB), Bucharest, Romania, in 2007 and 2011, respectively.

In 2010, she performed a six-month internship at the German Aerospace Center (DLR), Oberpfaffenhofen, Germany, as a visiting Ph.D. student in the Department of Earth Observation and Remote Sensing. Since 2007, she has been a Research Engineer with the Department of Applied Electronics and Information Engineering, Faculty of Electronics, Telecommunications and Information Technology (ETTI), UPB She took part in several research projects at national and international levels Her research fields include Earth Observation image processing and data mining based on information theory, information retrieval, data fusion, change detection, temporal analysis in image time series, and semantic annotation for very high resolution image understanding and interpretation.

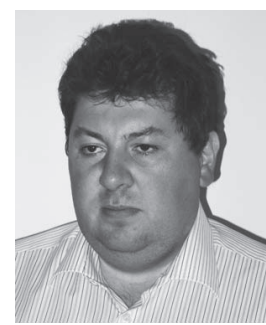

Teodor Costăchioiu received the B.S. and Ph.D. degrees in electronics and telecommunications from the University "Politehnica" of Bucharest (UPB), Bucharest, Romania, in 1997 and 2011, respectively.

In 2011, he performed a three-month internship at the German Aerospace Center (DLR), Oberpfaffenhofen, Germany, as a visiting Ph.D. student in the Remote Sensing Technology Institute (IMF). $\mathrm{He}$ is currently with the Department of Applied Electronics and Information Engineering, Faculty of Electronics, Telecommunications and Information Technology (ETTI), UPB. He published several papers concerning the analysis of satellite image time series and semantic analysis of Earth observation data. His interest is in embedded systems, Earth observation data processing, and time series analysis. 


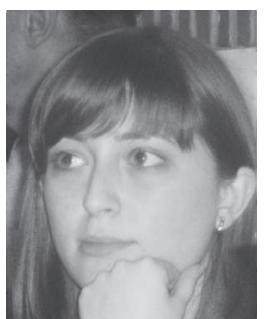

Carmen Pătraşcu was born in Craiova, Romania, in 1986. She received the B.S. degree in electrical engineering and computer science and the M.Sc. degree in advanced digital imaging from the Faculty of Electronics, Telecommunications and Information Technology (ETTI), University "Politehnica" of Bucharest (UPB), Bucharest, Romania, in 2009 and 2011, respectively, where she is currently working toward the Ph.D. degree.

Since 2009, she has been with the Department of Applied Electronics and Information Engineering, ETTI, UPB, as a Research Assistant in the frame of several research projects at national and international levels. Her primary interests are in synthetic aperture radar (SAR) signal processing, SAR interferometry and tomography, remote sensing, and image information mining.

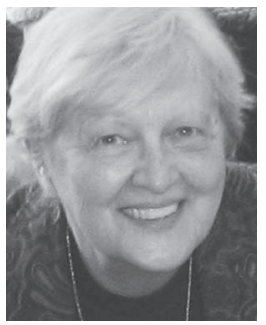

Inge Gavăt (SM'08) received the M.S. and Ph.D. degrees in electronics and telecommunications from the University "Politehnica" of Bucharest (UPB), Bucharest, Romania, in 1963 and 1998, respectively.

She is currently a Professor with the Department of Applied Electronics and Information Engineering, Faculty of Electronics, Telecommunications and Information Technology (ETTI), UPB, and also with the German Branch of the Faculty of Engineering in Foreign Languages, UPB. She is teaching courses in information and estimation theory, in communication theory, and in signal processing and artificial intelligence for man-machine communication. She is often a Visiting Professor at the Technische Universität Darmstadt, Darmstadt, Germany, and the University of Rouen, Rouen, France. She is involved in advanced research projects and programs in pattern recognition with the Romanian Academy of Sciences, Bucharest, the Military Technical Academy, Bucharest, the Romanian Space Agency, and the Ministry for Education and Research. She is also involved in bilateral international cooperation with the Technical University of Munich, Munich, Germany, University of Maribor, Maribor, Slovenia, and Rutgers University, Piscataway, N.J. She is developing algorithms for pattern recognition with Markov models, neural networks, and fuzzy systems. She worked on recognition applications, with emphasis on man-machine dialog and multimedia applications. In this field, she published numerous papers and a book, together with her research team. Recently, she has been involved in several projects in the frame of the FP6 Program concerning new technologies to support sustainable humanitarian crisis management using remote sensing imagery. She is also the Coordinator of several ongoing national projects developed in collaboration with the Romanian Space Agency, regarding data mining for floods and other anomaly detection. She has published several papers regarding the use of information theory in the field of data mining and semantic interpretation. Her interest is in stochastic processes conveying information, model-based sequence recognition and understanding, basics of man-machine communication, and information managing and retrieval in extended databases.

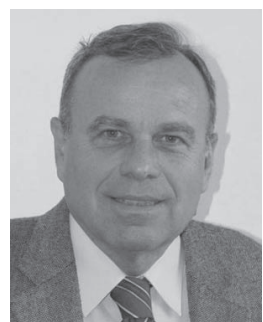

Vasile Lăzărescu (M'96) received the B.S. degree in electronic engineering from the University "Politehnica" of Bucharest (UPB), Bucharest, Romania, and the M.S. and Ph.D. degrees in electronic engineering from UPB in 1970 and 1983, respectively.

$\mathrm{He}$ is currently a Professor with the Department of Applied Electronics and Information Engineering, Faculty of Electronics, Telecommunications and Information Technology (ETTI), UPB. He has authored/coauthored 14 books and published more than 100 refereed journal and conference papers. His research and teaching interests include signal processing, computational intelligence, data acquisition and processing, and computer architecture.

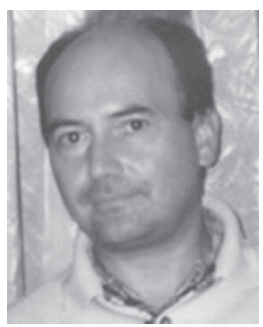

Mihai Datcu (SM'04) received the M.S. and Ph.D. degrees in electronics and telecommunications from the University "Politechnica" of Bucharest (UPB), Bucharest, Romania, in 1978 and 1986, respectively, and the title Habilitation á diriger des recherches in computer science from University Louis Pasteur. Strasbourg, France, in 1999.

Since 1981, he has been a Professor with the Faculty of Electronics, Telecommunications and Information Technology (ETTI), UPB, working on signal/image processing and electronic speckle interferometry, where has been the Director of the Research Center for Spatial Information since 2011. Since 1993, he has been a Scientist with the German Aerospace Center (DLR), Oberpfaffenhofen, Germany, where he is currently a Senior Scientist and an Image Analysis Research Group Leader in the Remote Sensing Technology Institute (IMF). He is developing algorithms for analyzing very high resolution synthetic aperture radar (SAR) and interferometric SAR data. He is engaged in research related to information theoretical aspects and semantic representations in advanced communication systems. Since 2011, he has also been leading the Immersive Visual Information Mining Research Laboratory, Munich Aerospace Faculty, Munich, Germany. He has held Visiting Professor appointments with the University of Oviedo, Oviedo, Spain; University Louis Pasteur; the International Space University, Strasbourg; the University of Siegen, Siegen, Germany; the University of Camerino, Camerino, Italy; and the Swiss Center for Scientific Computing (CSCS), Manno, Switzerland. From 1992 to 2002, he had a longer Invited Professor assignment with the Swiss Federal Institute of Technology, Eidgenoessische Technische Hochschule Zurich, Zurich, Switzerland. Since 2001, he has initiated and led the Competence Centre on Information Extraction and Image Understanding for Earth Observation at Telecom ParisTech, Paris, France, a collaboration of DLR with the French Space Agency (CNES). He has been a Professor holder of the DLR-CNES Chair at Telecom ParisTech. He and his team have developed and are currently developing the operational image information mining (IIM) processor in the payload ground segment systems for the German missions TerraSAR-X, TanDEM-X, and the ESA Sentinel 1 and 2. He is the author of more than 200 scientific publications, among them about 50 journal papers, and a book on number theory. His interests are in information and complexity theory, stochastic processes, Bayesian inference, and IIM.

Dr. Datcu is a member of the European Image Information Mining Coordination Group and the Data Archiving and Distribution Technical Committee of the IEEE Geoscience and Remote Sensing Society. 